$>$ Les signaux calciques sont organisés dans le temps et dans l'espace, ce qui leur permet d'assurer une signalisation cellulaire spécifique et robuste. En réponse à une stimulation, la concentration nanomolaire du signal $\mathrm{Ca}^{2+}$ peut augmenter de plusieurs dizaines de fois à proximité de quelques récepteurs de l'inositol (1, 4, 5) trisphosphate $\left(\operatorname{Ins}_{3}\right)$. Cela se fait sous la forme de vagues se propageant périodiquement dans un tissu ou un organe. Les études de la relation entre ces phénomènes, caractérisés par des échelles temporelles et spatiales très différentes, et les mécanismes qui en sont responsables, sont décrits dans cette revue par une approche fondée sur une interaction étroite entre expériences et modélisation, appliquée principalement à la signalisation calcique dans les hépatocytes. <

Jusqu'à très récemment, les biologistes mesuraient la concentration d'une protéine ou la vitesse de transformation d'un métabolite par exemple à partir de populations cellulaires. L'extraordinaire développement de nouvelles techniques d'imagerie (microscopie confocale, fluorescence resonance energy transfer [FRET], fluorescence recovery after photobleaching [FRAP], etc.), combiné à l'utilisation de sondes fluorescentes performantes, de protéines chimères (green fluorescent protein [GFP], DsRed, etc.) ou de nanocristaux (quantum dots) permet actuellement une approche quantitative et dynamique de la biologie d'une seule cellule, et rend possible l'analyse par exemple de l'expression spécifique d'un gène ou de la variation d'un flux métabolique. Ces approches révèlent dans bien des cas une assez grande hétérogénéité spatiale, mais aussi temporelle, rappelant, si besoin est, que les cellules sont des entités extrêmement dynamiques [1]. De manière plus inattendue, ces observations à l'échelle cellulaire mettent aussi en évidence le caractère aléatoire (stochastique) des événements moléculaires individuels. Le biologiste est dès lors confronté à de nouvelles questions : comment des événements qui se produisent sur

\section{Approches expérimentale et de modélisation de la signalisation calcique dans les cellules}

Laurent Combettes, Geneviève Dupont

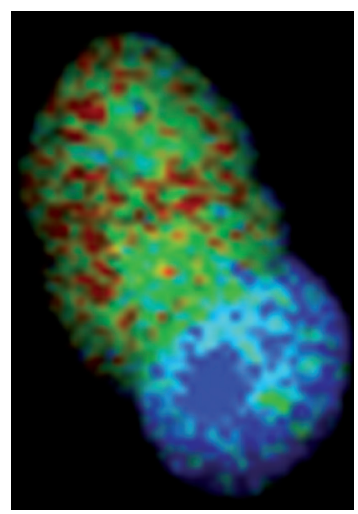

L. Combettes : UMR-S757, Inserm, Université Paris-Sud, Orsay, F-91405, France. G. Dupont : Unité de chronobiologie théorique, Université libre de Bruxelles, Faculté des sciences, Campus Plaine, boulevard du Triomphe, B-1050 Bruxelles, Belgique. gdupont@ulb.ac.be

des échelles de temps et d'espace très différentes sont-ils coordonnés? Comment les cellules contrôlent-elles, ou même utilisent-elles, des fluctuations moléculaires pour réaliser des tâches dites robustes? Pour répondre à ces questions, il est nécessaire d'associer démarches expérimentales et théoriques. Ce type d'approche pluridisciplinaire, utilisé avec succès depuis de nombreuses années en neurophysiologie, s'étend aujourd'hui à de nombreux aspects de la physiologie cellulaire. La signalisation calcique, objet de cette revue, en est un exemple frappant.

Depuis les travaux de Ringer il y a près de 130 ans, l'importance du calcium dans la physiologie cellulaire n'est plus à démontrer, mais l'extraordinaire complexité de l'organisation dans le temps et dans l'espace de cette signalisation n'a été révelée que grâce aux progrès techniques réalisés depuis une petite vingtaine d'années. En combinant expériences et modélisation, nous avons pu disséquer certains aspects de cette signalisation, notamment dans les hépatocytes. En réponse à une stimulation extracellulaire, les hépatocytes augmentent leur concentration en $\mathrm{Ca}^{2+}$ intracellulaire, celle-ci étant responsable de réponses physiologiques comme la production de glucose, la sécrétion biliaire, l'expression génétique ou la régénération du foie. Dans ces cellules, comme dans la plupart des cellules non excitables [2], des agonistes induisent la synthèse d'inositol $(1,4,5)$ trisphosphate $\left(\operatorname{Ins} P_{3}\right)$. L'Ins $P_{3}$ formé se fixe sur des récepteurs (Ins $\left.P_{3} R\right)$ /canaux calciques présents dans la membrane du réticulum endoplasmique (RE). La libération de $\mathrm{Ca}^{2+}$ via les récepteurs de l'Ins $\mathrm{P}_{3}$ est régulée par la concentration en $\mathrm{Ca}^{2+}$ dans le cytoplasme ; c'est aussi le cas pour les 
récepteurs de la ryanodine présents dans certains types cellulaires et dont les caractéristiques structurelles et fonctionnelles sont très proches de celles des $\operatorname{Ins}_{3} R[3,41]$. L'activité du canal est stimulée par de faibles concentrations de $\mathrm{Ca}^{2+}$ cytosolique, tandis que des concentrations plus élevées l'inhibent $[3,4]$. Cette propriété remarquable d'autorégulation biphasique par le $\mathrm{Ca}^{2+}$ de sa propre concentration cytoplasmique joue un rôle-clé dans l'organisation spatiotemporelle de ce messager. Comme $\mathrm{Ca}^{2+}$ et $\mathrm{Ins}_{3}$ sont requis pour ouvrir le canal, ces deux messagers sont qualifiés de co-agonistes du récepteur de l'Ins $P_{3}$. En outre, la libération de $\mathrm{Ca}^{2+}$ depuis les compartiments intracellulaires est généralement accompagnée par une stimulation de l'influx de $\mathrm{Ca}^{2+}$ depuis le milieu extracellulaire [2].

\section{Augmentations de $\mathrm{Ca}^{2+}$ localisées au niveau subcellulaire : blips, sparks, puffs}

Comme tous les canaux, les récepteurs-canaux sensibles à l'Ins $P_{3}$ s'ouvrent et se ferment de manière aléatoire en réponse à un faible taux de stimulation, provoquant de petites augmentations asynchrones de $\mathrm{Ca}^{2+}$. Les techniques de microscopie permettent maintenant d'observer de telles augmentations de $\mathrm{Ca}^{2+}$, qui correspondent à l'activité d'un ou deux récepteurs de l'Ins $P_{3}$. Ces événements microscopiques, qui provoquent des élévations de $\mathrm{Ca}^{2+}$ d'environ $40 \mathrm{nM}$ dans un volume d'l femtolitre et durent moins de $70 \mathrm{~ms}$, sont appelés blips dans les cellules non excitables [5]. Dans les cellules électriquement excitables, ces événements sont connus sous le nom de sparks et résultent de l'ouverture d'un petit nombre de récepteurs de la ryanodine [4]. Dans la cellule, les récepteurs ne sont pas distribués de manière homogène mais sont le plus souvent arrangés en clusters : ainsi le $\mathrm{Ca}^{2+}$ libéré par un récepteur de l'Ins $P_{3} R$ peut stimuler l'activité des autres récepteurs du cluster (Figure 1) puisque, comme nous l'avons vu plus haut, l'activité de ces canaux dépend du niveau de $\mathrm{Ca}^{2+}$ cytosolique. L'arrangement en clusters est indispensable car le $\mathrm{Ca}^{2+}$ est fortement tamponné dans le cytoplasme, ce qui empêche toute communication entre deux canaux distants de plus de quelques centaines de nanomètres. L'activation de l'ensemble des récepteurs de I'Ins $P_{3}$ d'un cluster provoque une augmentation de $\mathrm{Ca}^{2+}$ plus importante et plus longue (environ $170 \mathrm{nM}$ et $500 \mathrm{~ms}$ ) (appelée puff). Des simulations numériques de ces puffs, fondées sur les caractéristiques électrophysiologiques des récepteurs de l'Ins $\mathrm{P}_{3}$, suggèrent que 20 à 30 canaux sont nécessaires pour rendre compte des augmentations de $\mathrm{Ca}^{2+}$ observées in vivo [6]. Ces augmentations de $\mathrm{Ca}^{2+}$ sont suffisamment importantes pour provoquer l'activation de clusters proches permettant, dans certaines conditions, la transition entre signalisation calcique locale et globale [7] (Figure 1). Blips et puffs ne sont en effet observés qu'à de très faibles niveaux de stimulation, quand seulement quelques récepteurs ont fixé de l'InsP $P_{3}$ et sont donc activables. Pour des niveaux de stimulation plus élevés, tous les clusters participent à la dynamique et l'augmentation de $\mathrm{Ca}^{2+}$ se propage dans l'ensemble de la cellule, telle une vague calcique intracellulaire. Ces vagues parfois spectaculaires ${ }^{1}$ peuvent

${ }^{1}$ Voir par exemple : http://parkerlab.bio.uci.edu/images_movies_presentations/ calcium.htm.

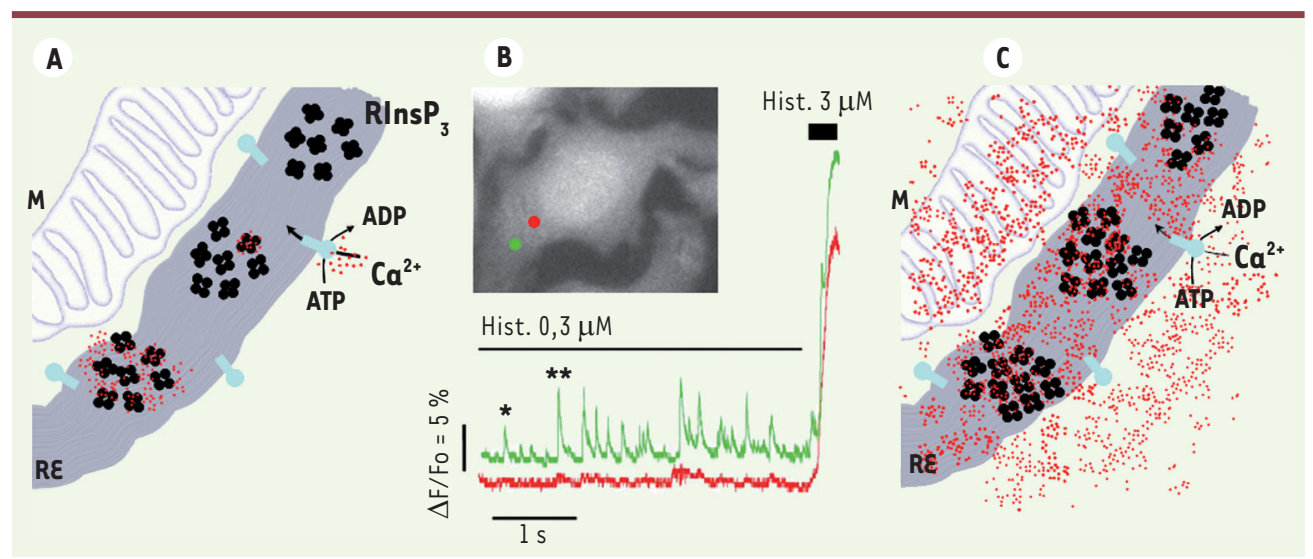

Figure 1. Augmentations locales de calcium. A. Représentation schématique de l'organisation en clusters des récepteurs de I'Ins $P_{3}$. La fixation de l'Ins $P_{3}$ sur son récepteur $\left(\operatorname{Ins} \mathrm{P}_{3} \mathrm{R}\right)$ provoque la libération de $\mathrm{Ca}^{2+}$ (points rouges) accumulé par les pompes calciques (bleu) dans le réticulum endoplasmique (RE). Les mitochondries (M) sont généralement localisées à proximité des récepteurs de l'Ins $\mathrm{P}_{3}$ et participent de manière active à la signalisation calcique. Lorsqu'un seul récepteur s'ouvre, l'augmentation de $\mathrm{Ca}^{2+}$ très localisée et fugace qui en résulte est appelée blip (* trace verte, panneau $B$ ). Pour une stimulation plus importante, plusieurs récepteurs de l'Ins $\mathrm{P}_{3}$ s'ouvrent, le $\mathrm{Ca}^{2+}$ ainsi libéré favorise l'ouverture des autres récepteurs présents dans le cluster, donnant naissance à un puff ( $* \star$ trace verte, panneau $B$ ). La mesure des variations de $\mathrm{Ca}^{2+}$ dans deux endroits proches du cytosol d'une cellule HeLa illustre bien ces phénomènes (panneau $B$, points vert et rouge). L'addition d'une faible concentration d'histamine (Hist. 0,3 $\mu \mathrm{M}$ ) provoque de petites augmentations ( $*$ blips, $\star \star *$ puffs) localisées dans la cellule (trace verte versus rouge, panneau $B$ ). Une forte concentration d'histamine $(3 \mu M)$ provoque une réponse globale de cette cellule (schématisée en $C$ ). Ces augmentations de $\mathrm{Ca}^{2+}$ globales se répètent dans le temps sous formes d'oscillations plus ou moins régulières dont la fréquence varie avec la force du stimulus. L'hépatocyte en est un exemple remarquable, qui montre des oscillations de Ca ${ }^{2+}$ régulières lorsqu'il est stimulé par des agonistes dépendant de l'Ins $\mathrm{P}_{3}$ tels que la noradrénaline ou la vasopressine (exemples dans la Figure 2A). 
adopter différentes formes. Le caractère aléatoire des canaux individuels y est masqué par la loi des grands nombres, et les modèles qui en rendent compte sont déterministes. Dans tous les cas, la modélisation montre que, quelle que soit leur forme, ces vagues sont dues à la propagation, dans un cytosol excitable, d'un pulse de calcium émis localement et de manière répétée [8]. Autrement dit, le calcium augmente dans une région spécifique de la cellule (parfois appelée site pacemaker) et active la libération de $\mathrm{Ca}^{2+}$ dans les régions adjacentes. Par exemple, lors de la fécondation, le $\mathrm{Ca}^{2+}$ augmente initialement autour du point de fixation du spermatozoïde, qui correspond à la zone de l'œuf où la concentration en Ins $_{3}$ est la plus élevée. Ce mécanisme de propagation sous forme de vagues est classique, aussi bien en chimie qu'en biologie.

\section{Oscillations de calcium au niveau cellulaire}

Il est maintenant bien connu que les augmentations de $\mathrm{Ca}^{2+}$ qui se propagent dans la cellule en réponse à une stimulation externe se présentent sous forme de pics répétés [2]. Ces pics, ou oscillations de $\mathrm{Ca}^{2+}$, sont principalement dus à la libération de $\mathrm{Ca}^{2+}$ depuis le réticulum endoplasmique (Figure 1 ). De nombreux modèles théoriques ont montré que les caractéristiques de la régulation des $\operatorname{Ins}_{3} R$, qui sont soumis à un rétrocontrôle à la fois positif et négatif par le $\mathrm{Ca}^{2+}$ (voir plus haut), sont responsables de la nature oscillatoire des signaux calciques. Ce même mécanisme fondamental s'applique à la très grande majorité des types cellulaires.

\section{Trois types de récepteurs de l'Ins $\mathbf{P}_{3}$}

Les caractéristiques détaillées de ces oscillations dépendent toutefois du type cellulaire et de l'agoniste, et pour expliquer ces spécificités, il faut introduire dans ce schéma de régulation globale plusieurs éléments distinctifs. Une différence majeure est liée à l'existence de trois types d'Ins $P_{3} R$ [9], qui diffèrent par leur affinité pour l'Ins $P_{3}$ : le type 2 a la plus grande affinité et le type 3 la plus faible. De plus les trois types d'Ins $P_{3} R$ sont régulés différemment par le $\mathrm{Ca}^{2+}$. Par exemple, le type 3 pourrait ne pas être inhibé par le $\mathrm{Ca}^{2+}$ au moins pour des concentrations de $\mathrm{Ca}^{2+}$ physiologiques [10]. Ces différences sont importantes pour le décours des signaux calciques. Ainsi, des expériences dans lesquelles les niveaux d'expression des trois formes $d^{\prime}$ Ins $P_{3} R$ sont modifiés (surexpression ou inhibition), montrent que les proportions des trois types de récepteurs Ins $_{3}$ affectent profondément le décours des signaux calciques [11]. Les modélisations mathématiques que nous avons réalisées rendent compte de ces observations et suggèrent que le récepteur $\operatorname{lns} \mathrm{P}_{3}$ de type 2, qui présente la dépendance la plus sensible au $\mathrm{Ca}^{2+}$, est l'oscillateur principal. Autrement dit, plus une cellule exprime de récepteurs de l'Ins $\mathrm{P}_{3}$ de type 2 , plus elle aura tendance à présenter des oscillations de $\mathrm{Ca}^{2+}$. La stimulation de l'Ins $P_{3} R$ de type 1 induit aussi des oscillations, mais plus irrégulières et moins soutenues. L'activation du récepteur de type 3 , quant à elle, tend à supprimer les oscillations. Cet effet inattendu s'explique par le comportement de l'Ins $\mathrm{P}_{3} \mathrm{R}$ de type 3, qui n'est pas inhibé par le $\mathrm{Ca}^{2+}$, et permet une sortie constante de $\mathrm{Ca}^{2+}$ qui va inhiber les deux autres récepteurs. Cependant, comme le montrent les simulations, cet effet ne se produit que pour de fortes densités d'Ins $P_{3} R$. Au contraire, pour de faibles densités, le flux constant de $\mathrm{Ca}^{2+}$ induit par l'ouverture de l'Ins $P_{3} R$ de type 3 pourrait stimuler l'activation des deux autres types de récepteurs de l'Ins $\mathrm{P}_{3}$ et favoriserait donc l'apparition des oscillations [12].

\section{Régulation par le $\mathrm{Ca}^{2+}$ du métabolisme de l'Ins $\mathrm{P}_{3}$}

Comme d'autres phénomènes oscillants, rythmes circadiens ou cycle cellulaire par exemple, les oscillations de $\mathrm{Ca}^{2+}$ sont soumises à des mécanismes de régulation qui, sans vraiment affecter leur existence, peuvent influencer significativement leurs caractéristiques. L'effet du $\mathrm{Ca}^{2+}$ sur le métabolisme de l'InsP $\mathrm{P}_{3}$ en est un exemple: la concentration de $\mathrm{Ca}^{2+}$ intracellulaire peut en effet affecter à la fois la synthèse et la dégradation d'Ins $P_{3}$ $[13,14]$. Cependant, alors que la stimulation par le $\mathrm{Ca}^{2+}$ de la production d'Ins $\mathrm{P}_{3}$ est un mécanisme qui peut générer des oscillations même en l'absence de régulation de l'Ins $\mathrm{P}_{3} \mathrm{R}$ par le $\mathrm{Ca}^{2+}[15,16]$, la stimulation de la dégradation d'Ins $\mathrm{P}_{3}$ par le $\mathrm{Ca}^{2+}$ induit simplement des oscillations passives d'Ins $P_{3}$ qui affectent peu les oscillations de $\mathrm{Ca}^{2+}$ [17]. La distinction entre ces deux types d'oscillations peut être identifiée en suivant les variations du niveau d'Ins $\mathrm{P}_{3}$ pendant les oscillations de $\mathrm{Ca}^{2+}$. Les résultats obtenus montrent que les situations varient en fonction du type cellulaire étudié [18]. En outre, des approches de modélisation ont établi que la réponse d'une cellule présentant des oscillations de $\mathrm{Ca}^{2+}$ à un pulse supplémentaire d'InsP $\mathrm{P}_{3}$ est qualitativement différente selon que les oscillations de $\mathrm{Ca}^{2+}$ entraînent celles d'Ins $\mathrm{P}_{3}$ ou vice-versa [19].

\section{Deux scénarios}

pour la propagation spatiale des vagues de $\mathrm{Ca}^{2+}$

Du point de vue spatial, les oscillations de $\mathrm{Ca}^{2+}$ correspondent en réalité à des ondes qui se propagent de manière périodique dans le cytoplasme. Tout se passe comme si, à partir d'un certain niveau d'Ins $P_{3}$, les augmentations locales de $\mathrm{Ca}^{2+}$ (blips et puffs) devenaient suffisamment fréquentes et importantes pour envahir tout le cytoplasme sous forme de vagues, et qu'en plus, cette augmentation globale se produisait alors de manière régulière dans le temps (Figure 2 A3 pour exemple). La transition entre ces deux régimes microscopique et aléatoire d'une part, et cellulaire et régulier d'autre part - observée en réponse à la simple augmentation de la stimulation cellulaire reste mal comprise par les théoriciens. Deux scénarios sont en effet possibles. Selon le premier, la transition puffs-oscillations serait due à un processus dit de nucléation: 
dans cette hypothèse, étant donné la mauvaise communication entre clusters, une vague ne peut être déclenchée que si, par hasard, plusieurs sites puffs voisins sont actifs en même temps. L'augmentation de $\mathrm{Ca}^{2+}$ qui en résulte est suffisamment importante pour activer de proche en proche l'ensemble des récepteurs à I'Ins $P_{3}$ de la cellule. La relative régularité de ce processus d'initiation serait due au nombre élevé de sites puffs fonctionnels (c'est-à-dire ayant lié l'Ins $P_{3}$ ) présents dans la cellule, mais la description déterministe ne serait pas valable. En effet, la mauvaise communication entre les sites puffs ne permet pas de décrire correctement la dynamique en termes de concentration moyenne en $\mathrm{Ca}^{2+}[20]$. Selon l'autre scénario, la transition puffs-oscillations résulterait simplement de l'augmentation du nombre de récepteurs de l'Ins $P_{3}$ fonctionnels. Celle-ci améliore la communication entre les sites puffs tandis que l'impact des fluctuations moléculaires s'amenuise car, en moyenne, les fluctuations se compensent et la dynamique calcique correspond à un comportement

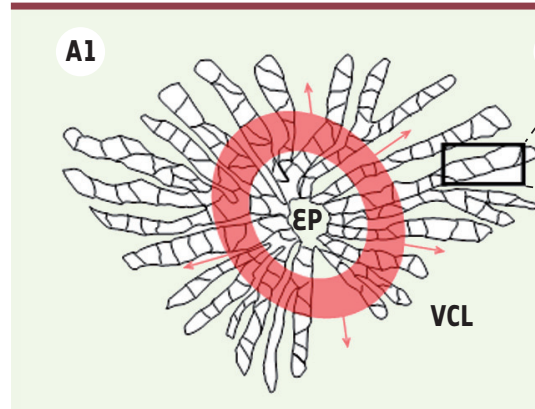

A3

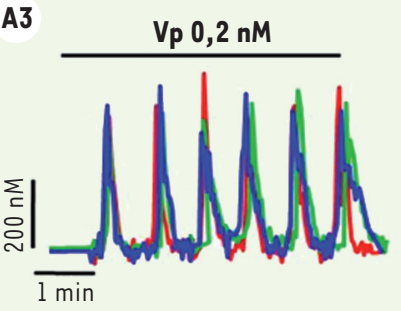

B1

\begin{tabular}{|c|c|c|}
\hline $\begin{array}{l}\text { Récep } \\
\text { de l'l }\end{array}$ & $\mathrm{IP}_{3} \mathrm{R}_{\mathrm{INH}} \stackrel{\mathrm{Ca}^{2+}>500 \mathrm{nM}}{\stackrel{\mathrm{Ca}^{2+}<500 \mathrm{nM}}{\rightleftarrows}}$ & $\frac{d R_{I N H}}{d t}=k+C^{n i} \frac{K_{A}^{n a}}{K_{A}^{n a}+C^{n a}}-k_{-} R_{I N H}$ \\
\hline$\left[\mathrm{Ca}^{2+}\right]_{\mathrm{cyt}}$ & $\mathrm{C}_{\mathrm{RE}} \stackrel{\text { POMPE }}{\longrightarrow} \mathrm{C}$ & $\frac{d C}{d t}=k_{1}\left(b+I P_{3} R_{\text {ouvert }}\right)\left(C-C_{R \varepsilon}\right)-V_{M P} \frac{C^{2}}{C^{2}+K_{P}^{2}}$ \\
\hline$\left[\operatorname{Ins} P_{3}\right]$ & 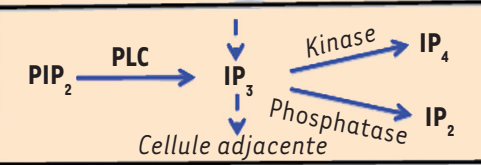 & $\begin{array}{l}\frac{d I P_{3}}{d t}=V_{p l c}-V_{K} \frac{I P_{3}}{I P_{3}+K_{K}} \frac{C^{n}}{C^{n}+K_{2}^{n}} \\
-V_{P} \frac{I P_{3}}{I P_{3}+K_{p}}+P\left(I P_{3}^{(i+1)}+I P_{3}^{(i-1)}-2 I P_{3}^{(i)}\right)\end{array}$ \\
\hline
\end{tabular}

B2

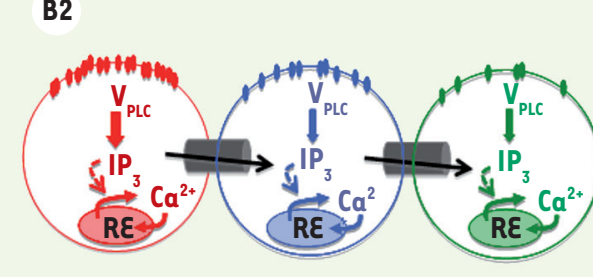

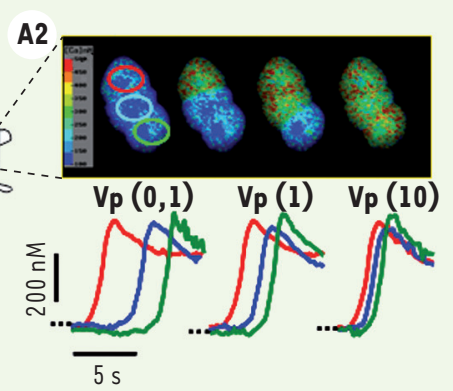

A4

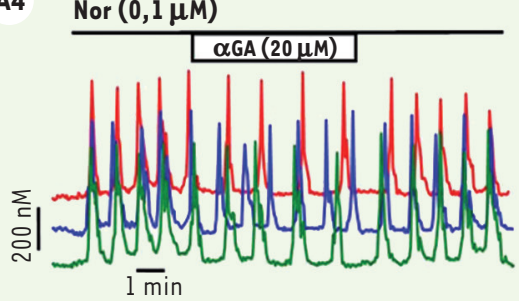

Figure 2. Vagues calciques. A. Vagues calciques dans le foie. La perfusion, dans des foies entiers (schématisés en $\boldsymbol{A l}$ ) ou sur des triplets d'hépatocytes (A2), d'agonistes dépendants de l'InsP $P_{3}$ tels que la vasopressine $(\mathrm{Vp})$ ou la noradrénaline (Nor) provoque des augmentations de $\mathrm{Ca}^{2+}$ séquentielles donnant l'apparence de vagues calciques intercellulaires. Dans le foie, ces vagues sont unidirectionnelles et se propagent de l'espace porte $(\varepsilon P)$ vers la veine centro-lobulaire ( $\mathrm{VCL}$ ) des lobules (A1). Les trois traces de différentes couleurs représentent les mesures de $\mathrm{Ca}^{2+}$ dans les différentes cellules d'un triplet d'hépatocytes et l'image montre les cellules avant (en bleu) et après (en vert) l'augmentation de la concentration en $\mathrm{Ca}^{2+}$ (A2). Les traces montrent que, quelle que soit la concentration de l'agoniste (ici vasopressine Vp de 0,1 à $10 \mathrm{nM}$ ), l'ordre de la séquence des réponses reste le même, seul le délai intercellulaire diminue. Ces vagues apparentes de $\mathrm{Ca}^{2+}$ se répètent dans le temps donnant naissance à des oscillations calciques coordonnées (A3). L'effet d'une inhibition des jonctions communicantes par de l' $\alpha$-glycyrrhetinic acid $(\alpha G A, 20 \mu M)$ montre l'importance de ces jonctions pour assurer la coordination de ces oscillations calciques (A4). B. Modélisation des vagues calciques se propageant dans des fragments de travées hépatocytaires. Le modèle est fondé sur la description de la dynamique calcique intracellulaire par trois variables (fraction de récepteurs de l'Ins $P_{3}$ dans un état inhibé, concentration en $\mathrm{Ca}^{2+}$ cytosolique et concentration en $\left.\operatorname{lns} \mathrm{P}_{3}\right)$. Les processus de régulation, transport et métabolisme dans lesquels ces variables sont impliquées sont représentés dans la colonne centrale du panneau $B 1$ et les équations d'évolution correspondantes dans la colonne de droite. Pour modéliser la dynamique d'un triplet d'hépatocytes, trois systèmes semblables d'équations sont couplés, via le passage d'Ins $\mathrm{P}_{3}$ d'une cellule à l'autre (B2). Les cellules diffèrent par leur nombre de récepteurs hormonaux, et donc par leur vitesse de synthèse $\mathrm{d}^{\prime} \mathrm{InS}_{3}$. Le panneau $B 3$ représente les résultats d'une simulation du modèle : la cellule qui est la plus sensible à I’hormone (rouge) présente le pic de $\mathrm{Ca}^{2+}$ en premier lieu. Les pics de $\mathrm{Ca}^{2+}$ sont ensuite émis de manière répétitive et séquentielle par les cellules adjacentes (bleue puis verte, $\mathbf{B 2}$ ), donnant l'apparence d'un processus de propagation d'onde. 
périodique déterministe. Cette deuxième hypothèse est corroborée par une confrontation entre résultats expérimentaux et simulations stochastiques: celle-ci montre que, dans les hépatocytes, le niveau d'irrégularité des oscillations peut être relié au nombre de récepteurs à l'InsP $P_{3}[21]$.

\section{Rôle physiologique des ondes de $\mathrm{Ca}^{2+}$}

Bien que les mécanismes qui engendrent les oscillations et ondes de $\mathrm{Ca}^{2+}$ commencent à être bien connus, le rôle physiologique de ces oscillations reste beaucoup moins bien documenté. Dès leur mise en évidence, ces signaux ont été proposés comme un exemple de codage par fréquence de l'activation cellulaire; on sait que ce type de codage est plus résistant au «bruit $»^{2}$ que le codage en amplitude [22]. Un tel codage par fréquence pourrait impliquer la CaMKII $\left(\mathrm{Ca}^{2+} / \mathrm{calmodu-}\right.$ lin-dependent protein kinase $/ I$ ), une enzyme ubiquitaire dont le taux d'activité dépend de la fréquence des oscillations de $\mathrm{Ca}^{2+}$ [23]. Cette kinase, constituée de 10 à 12 sous-unités, possède la particularité de pouvoir s'autophosphoryler une fois qu'un nombre suffisant de monomères ont fixé le complexe $\mathrm{Ca}^{2+}$-calmoduline. Dans cet état, la CaMKII, dite autonome, garde son activité de kinase, même en l'absence de $\mathrm{Ca}^{2+}$. Des pics de $\mathrm{Ca}^{2+}$ suffisamment rapprochés permettent de déclencher cette activité autonome. En revanche, un intervalle de temps trop important entre deux pics de $\mathrm{Ca}^{2+}$ laisse au complexe $\mathrm{Ca}^{2+}$-calmoduline le temps de se dissocier, empêchant l'autophosphorylation [24].

II peut y avoir d'autres avantages physiologiques à un signal oscillant de $\mathrm{Ca}^{2+}$ que le seul codage de fréquence, notamment pour éviter les effets délétères d'une augmentation soutenue de $\mathrm{Ca}^{2+}$ [25]. Des oscillations de $\mathrm{Ca}^{2+}$ variant à la fois en amplitude, en durée et en fréquence sont ainsi capables d'activer des ovocytes non fécondés de mammifères [26], certains profils d'augmentations calciques semblant plus efficaces que d'autres pour engager les premières divisions du développement embryonnaire [27]. Dans d'autres cellules, par exemple les lymphocytes $B$, les oscillations permettent de sensibiliser les cellules à de faibles taux de stimulation [28].

\section{Coordination des signaux $\mathrm{Ca}^{2+}$ au niveau supracellulaire}

Des observations datant de plus de 20 ans, faites dans des expériences réalisées notamment sur des foies entiers perfusés, suggèrent une organisation des signaux calciques dans l'espace et dans le temps au niveau d'un tissu entier [29]. Plus récemment, il a été montré dans certains organes ou dans des systèmes multicellulaires que les vagues calciques intracellulaires observées dans une cellule se propageaient aux cellules adjacentes, que ces dernières soient de même type ou d'un type différent [18]. Par exemple, dans des expériences utilisant des tranches d'hypothalamus de rat, une activité calcique spontanée et coordonnée peut être observée dans les astrocytes [30]. Cette activité peut même se transmettre à un autre type cellulaire : la stimulation électrique de certains neurones peut induire des vagues calciques dans les astrocytes adjacents [31]. Différents mécanis-

${ }^{2}$ Bruit est ici pris dans le sens de bruit moléculaire des systèmes biologiques. mes, non exclusifs, expliquent ces observations. Citons les jonctions communicantes (gap junctions) qui permettent la diffusion de petites molécules entre cellules connectées, et dont le rôle a été très bien étudié [18]. Dans la plupart des cas, les vagues intercellulaires résultent de la diffusion $d^{\prime}$ Ins $P_{3}$ d'une cellule à une autre. Certaines observations suggèrent que d'autres messagers intracellulaires tels que I'ADP ribose ou le $\mathrm{Ca}^{2+}$, pourraient participer à ces vagues calciques [18]. Quel que soit le messager, les vagues intercellulaires qui ne sont portées que par leur diffusion au travers des jonctions communicantes sont limitées dans le temps et dans l'espace, ne se propageant qu'à quelques cellules adjacentes. Dans les tissus, qui comportent un nombre beaucoup plus élevé de cellules, un mécanisme de régénération de ces vagues est nécessaire pour permettre leur propagation. Le plus commun fait intervenir la voie de synthèse de l'Ins $\mathrm{P}_{3}$ (voir ci-dessus), via la libération dans le milieu extracellulaire de molécules capables d'activer cette voie de signalisation. En fonction des types cellulaires étudiés, I'ATP, le glutamate ou encore le monoxyde d'azote (NO) sont ainsi les molécules le plus souvent impliquées dans la propagation des vagues calciques intercellulaires [18]. En fait, des études réalisées sur des cellules en culture montrent que des voies paracrines et directes (jonctions communicantes) s'associent pour assurer la propagation des signaux calciques intercellulaires. C'est sans doute aussi le cas in vivo, bien qu'il soit difficile de l'étudier.

L'importance relative de ces deux voies peut varier en fonction des situations physiologiques. Le foie nous en offre un exemple remarquable. Dans le foie normal, la perfusion d'agonistes dépendants $\mathrm{du} \mathrm{Ca}^{2+}$, vasopressine ou noradrénaline, provoque des vagues de $\mathrm{Ca}^{2+}$ répétitives qui se propagent de façon synchronisée aux différents lobules constitutifs du foie [32]. Des expériences réalisées sur des fragments de travées hépatocytaires, dans lesquels les hépatocytes restent connectés par leurs jonctions communicantes, montrent que ces vagues de $\mathrm{Ca}^{2+}$ ne sont qu'apparentes et résultent d'un léger décalage de phase d'oscillations dont les fréquences sont très proches (Figure 2A). Ce décalage de phase est dû à un gradient de récepteurs de la vasopressine ou de la noradrénaline le long des travées d'hépatocytes, lui-même responsable d'un gradient de vitesses d'activation de la phospholipase $C$ (PLC) (Figure 2B). Les vitesses de synthèse d'Ins $\mathrm{P}_{3}$ sont donc légèrement différentes d'une cellule à l'autre [32, 33]. En conséquence, les pics d'Ins $\mathrm{P}_{3}$, et donc de $\mathrm{Ca}^{2+}$, apparaissent séquentiellement le long des travées. La coordination de ces oscillations est quant à elle assurée par la diffusion de faibles quantités d'Ins $P_{3}$ à travers les jonctions communicantes [34]. Cette quantité de messagers n'est cependant pas suffisante à elle seule pour déclencher des réponses en $\mathrm{Ca}^{2+}[34,35]$. Un modèle fondé sur ces observations nous a 
permis de formaliser ces hypothèses (Figure 2B) et de faire certaines prédictions qui ont pu être vérifiées expérimentalement [33]. Le modèle décrit simplement les évolutions temporelles du $\mathrm{Ca}^{2+}$, de $\mathrm{I}^{\prime} \mathrm{Ins}_{3}$ et de la fraction de récepteurs de l'InsP $\mathrm{P}_{3}$ qui sont dans un état inactif (car inhibés par des taux élevés de $\mathrm{Ca}^{2+}$ cytosolique). Le passage d'Ins $\mathrm{P}_{3}$ à travers les jonctions communicantes y est également décrit, la perméabilité de ces dernières apparaissant dès lors comme un paramètre-clé du modèle [33].

\section{Un exemple d'implications physiologiques : signaux $\mathrm{Ca}^{2+}$ et fonction hépatique}

L'ensemble de ces résultats nous a permis de proposer un modèle d'organisation spatiotemporelle du signal $\mathrm{Ca}^{2+}$ dans le foie qui pourrait être important pour certaines des fonctions majeures de cet organe. Dès le début des années 1990, le groupe de Jungermann montrait que l'inhibition des jonctions communicantes inhibait la libération de glucose induite par une stimulation des nerfs sympathiques [36]. Ces résultats ont été confirmés en utilisant des souris n'exprimant pas la connexine 32, majoritaire dans les hépatocytes [18]. La communication intercellulaire assurée par les jonctions communicantes est aussi impliquée dans la sécrétion biliaire régulée par les agonistes dépendant $d u \mathrm{Ca}^{2+}$. Dans ce cas, l'importance de la vague unidirectionnelle de $\mathrm{Ca}^{2+}$ a aussi pu être mise en évidence : la suppression du gradient de sensibilité à la vasopressine, qui entraîne l'abolition du caractère séquentiel des réponses calciques, inhibe le flux biliaire stimulé par cet agoniste [32].

Un aspect fascinant du foie est sa capacité à se régénérer après une destruction partielle. Étant donné le rôle $\mathrm{du} \mathrm{Ca}^{2+}$ dans la régulation de la transcription des gènes et du cycle cellulaire, il n'est pas étonnant que la signalisation calcique joue là aussi un rôle majeur. Les expériences d'hépatectomie partielle chez le rat indiquent que de nombreuses molécules capables de provoquer des augmentations de $\mathrm{Ca}^{2+}$ sont libérées (notamment I'ATP [37]) et induisent ainsi la propagation de vagues calciques dans le foie. Il existe en outre une profonde réorganisation des signaux calciques intra- et intercellulaires au cours de la régénération hépatique [32]. Vingtquatre heures après hépatectomie partielle, il y a une désensibilisation de la signalisation calcique dans les hépatocytes qui résulte notamment d'une modification de l'expression des récepteurs de I'InsP $P_{3}[38,39]$. Avant l'intervention, les hépatocytes expriment les récepteurs de l'Ins $\mathrm{P}_{3}$ de type 1 et 2 (environ $30 \%$ et $70 \%$ respectivement). Vingt-quatre heures après hépatectomie partielle, le nombre total de récepteurs de l'Ins $\mathrm{P}_{3}$ diminue très fortement et la proportion relative des récepteurs de type 1 et 2 s'équilibre. Parallèlement on observe une modification de la forme des oscillations de $\mathrm{Ca}^{2+}$ induites par les agonistes dépendants de l'Ins $\mathrm{P}_{3}$, notamment la vasopressine [39]. Récemment, en interférant directement avec la signalisation $\mathrm{du} \mathrm{Ca}^{2+}$ intracellulaire, nous avons pu confirmer l'importance des variations de $\mathrm{Ca}^{2+}$ cytosolique pour le processus de régénération du foie. On observe ainsi, une heure après hépatectomie partielle, une diminution de l'induction du gène précoce $c$-fos et un défaut de phosphorylation d'ERK1/2 (extracellular signal-regulated kinase) lorsque le $\mathrm{Ca}^{2+}$ cytosolique est chélaté. L'ensemble des résultats obtenus montre que, dans les hépatocytes, le $\mathrm{Ca}^{2+}$ cytosolique joue un rôle positif dans les phases précoces du cycle cellulaire, notamment lors de la transition $\mathrm{GO} / \mathrm{Gl}$ et de la progression vers la phase S [40]. À ce stade, on peut à nouveau s'attendre à ce qu'une approche de modélisation, fondée sur une description couplée des oscillations de $\mathrm{Ca}^{2+}$ et du cycle cellulaire, favorise une compréhension globale et quantitative de ce phénomène fascinant. $\diamond$

\section{SUMMARY}

Experimental and computational

approach of calcium signaling

In many cell types, specific and robust signalling relies on a high level of spatiotemporal organization of $\mathrm{Ca}^{2+}$ dynamics. In response to external stimulation, $\mathrm{Ca}^{2+}$ signals ranging from a small increase of a few tens of nanomolar concentrations at the mouth of an inositol 1, 4, 5-trisphosphate receptor to the periodic propagation of waves invading an organ or a tissue, can be observed. Here, we review our combined experimental and computational approach of $\mathrm{Ca}^{2+}$ dynamics, which has been mainly carried out on liver hepatocytes. We focus in particular on the understanding of the relationship between elementary $\mathrm{Ca}^{2+}$ increases, $\mathrm{Ca}^{2+}$ oscillations and intra- or intercellular $\mathrm{Ca}^{2+}$ waves. The physiological impact of such signalling on liver function is also discussed. $\diamond$

\section{REMERCIEMENTS}

Laurent Combettes reçoit le soutien de l'ANR (projet RPV07094LSA) et d'un PNR en hépato-gastro-entérologie. Il bénéficie d'un contrat d'interface entre l'Inserm et l'AP-HP (Hôpital du Kremlin-Bicêtre). Geneviève Dupont est maître de recherche du FNRS belge et bénéficie d'un financement du Fonds de la recherche scientifique médicale (convention $n^{0} 3.4568 .10$ ) et d'un programme dans le cadre des pôles d'attraction interuniversitaire (projet P6/25). Cette collaboration est financée par un programme Tournesol PHC.

\section{CONFLIT D'INTÉRÊTS}

Les auteurs déclarent n'avoir aucun conflit d'intérêts concernant les données publiées dans cet article.

\section{RÉFÉRENCES}

1. Goldbeter A, Gérard C, Leloup JC. Biologie des systèmes et rythmes cellulaires. Med Sci (Paris) $2010 ; 26: 49-56$

2. Berridge $M$, Bootman $M$, Roderick H. Calcium signaling: dynamics, homeostasis and remodeling. Nat Rev Mol Cell Biol $2003 ; 1: 11-21$.

3. Kushnir A, Betzenhauser M, Marks A. Ryanodine receptor studies using genetically engineered mice. FEBS Lett 2010 ; 584 : 1956-65.

4. Bezprozvanny I, Watras J, Ehrlich B. Bell-shaped calcium responses of $\operatorname{InSP}_{3}$ - and calcium-gated channels from endoplasmic reticulum of cerebellum. Nature 1991 ; $351: 751-4$.

5. Marchant J, Parker I. Role of elementary $\mathrm{Ca}^{2+}$ puffs in generating repetitive $\mathrm{Ca}^{2+}$ oscillations. EMBO J $2001 ; 20: 65-76$.

6. Swillens S, Dupont G, Combettes L, Champeil P. From calcium blips to calcium puffs: theoretical analysis of the requirements for interchannel communication. Proc Natl Acad Sci USA 1999 ; 96 : 13750-5.

7. Shuai J, Jung P. Optimal ion channel clustering for intracellular calcium signaling. Proc Natl Acad Sci USA 2003 ; 100 : 506-10.

8. Lechleiter J, Clapham D. Molecular mechanisms of intracellular calcium excitability in Xenopus laevis oocytes. Cell $1992 ; 69: 283-94$.

9. Foskett JK, White C, Cheung KH, Mak DO. Inositol trisphosphate receptor $\mathrm{Ca}^{2+}$ release channels. Physiol Rev $2007 ; 87$ : 593-658. 
10. Hagar RE, Burgstahler AD, Nathanson MH, Ehrlich BE. Type III InsP3 receptor channel stays open in the presence of increased calcium. Nature $1998 ; 396: 81-4$.

11. Miyakawa T, Maeda A, Yamazawa T, et al. Encoding of $\mathrm{Ca}^{2+}$ signals by differential expression of $\mathrm{IP}_{3}$ receptor subtypes. EMBO J $1999 ; 18: 1303-8$.

12. Dupont G, Combettes L. Modelling the effect of specific inositol 1, 4, 5 trisphosphate receptor isoforms on cellular $\mathrm{Ca}^{2+}$ signals. Biol Cell $2006 ; 98: 171-82$.

13. Rhee S Regulation of phosphoinositide-specific phospholipase C. Annu Rev Biochem $2001 ; 70$ : 281-312.

14. Takazawa M, Lemos M, Delvaux A, et al. Rat brain inositol 1, 4, 5-trisphosphate 3-kinase. Ca $^{2+}$ sensitivity, purification and antibody production. Biochem J $1990 ; 268: 213-7$.

15. Meyer T, Stryer L. Calcium spiking. Annu Rev Biophys Biophys Chem 1991; 20 : 153-74.

16. Kummer U, Olsen L, Dixon C, et al. Switching from simple to complex oscillations in calcium signaling. Biophys J $2000 ; 79: 1188-95$

17. Dupont $\mathrm{G}$, Koukoui $\mathrm{O}$, Clair $\mathrm{C}$, et al. $\mathrm{Ca}^{2+}$ oscillations in hepatocytes do not require the modulation of InsP 3-kinase activity by $\mathrm{Ca}^{2+}$. FEBS Lett $2003 ; 534: 101-5$.

18. Dupont $G$, Combettes L, Leybaert L. Calcium dynamics: spatio-temporal organization from the subcellular to the organ level. Int Rev Cytol 2007 ; 261 : 193-245.

19. Sneyd J, Tsaneva-Atanasova K, Reznikov V, et al. A method for determining the dependence of calcium oscillations on inositol trisphosphate oscillations. Proc Natl Acad Sci USA $2006 ; 103$ : 1675-80.

20. Falcke M Reading the patterns in living cells: the physics of $\mathrm{Ca}^{2+}$ signaling. Adv Physics $2004 ; 53: 255-440$.

21. Dupont G, Abou-Lovergne A, Combettes L. Stochastic aspects of oscillatory $\mathrm{Ca}^{2+}$ dynamics in hepatocytes. Biophys J 2008 ; 95 : 2193-202.

22. Rapp P. Why are so many biological systems periodic? Progr Neurobiol $1987 ; 29: 261-73$.

23. De Koninck P, Schulman H. Sensitivity of CaM kinase II to the frequency of $\mathrm{Ca}^{2+}$ oscillations. Science 1998 ; $279: 227-30$.

24. Dupont G, Houart P, De Koninck P. Sensitivity of CaM kinase II to the frequency of $\mathrm{Ca}^{2+}$ oscillations: a simple model. Cell Calcium $2003 ; 34: 485-97$.

25. Orrenius S, Nicotera P. The calcium ion and cell death. J Neural Transm $1994 ; 43$ (suppl) : 1-11.

26. Toth $S$, Huneau D, Banrezes B, Ozil JP. Egg activation is the result of calcium signal summation in the mouse. Reproduction $2006 ; 131: 27-34$.

27. Dupont $\mathrm{G}$, Heytens $\varepsilon$, Leybaert L. Oscillatory $\mathrm{Ca}^{2+}$ dynamics and cell cycle resumption at fertilization in mammals: a modelling approach. Int J Dev Biol $2010 ; 54: 655-65$.

28. Dolmetsch R, Xu K, Lewis R. Calcium oscillations increase the efficiency and specificity of gene expression. Nature $1998 ; 392: 933-8$.
29. Graf $P$, vom Dahl S, Sies H. Sustained oscillations in extracellular calcium concentrations upon hormonal stimulation of perfused rat liver. Biochem J 1987 ; 241 : 933-6.

30. Parri H, Gould T, Crunelli V. Spontaneous astrocytic $\mathrm{Ca}^{2+}$ oscillations in situ drive NMDAR-mediated neuronal excitation. Nat Neurosci $2001 ; 4$ : 803-12.

31. Fellin T, Sul J, D’Ascenzo M, et al. Bidirectional astrocyte-neuron communicaion: the many roles of glutamate and ATP. Novartis Found Symp $2006 ; 27: 208-17$.

32. Tordjmann T. Calcium signaling. In : Dufour JF, Clavien PA, eds. Signaling pathways in liver diseases. Berlin-Heidelberg : Springer-Verlag, 2009: 455-64.

33. Dupont G, Tordjmann T, Clair C, et al. Mechanism of receptor-oriented intercellular calcium wave propagation in hepatocytes. FASEB J $2000 ; 14: 279-89$.

34. Clair C, Chalumeau C, Tordjmann T, et al. Investigation of the roles of $\mathrm{Ca}^{2+}$ and Ins $\mathrm{P}_{3}$ diffusion in the coordination of $\mathrm{Ca}^{2+}$ signals between connected hepatocytes. J $\mathrm{Cell} \mathrm{Sci}$ $2001 ; 114: 1999-2007$

35. Tordjmann T, Berthon B, Claret M, Combettes L. Coordinated intercellular calcium waves induced by noradrenaline in rat hepatocytes: dual control by gap junction permeability and agonist. EMBO J $1997 ; 16: 5398-407$.

36. Seseke FG, Gardemann A, Jungermann K. Signal propagation via gap junctions, a key step in the regulation of liver metabolism by the sympathetic hepatic nerves. FEBS Lett $1992 ; 301: 265-70$.

37. Gonzales $\varepsilon$, Julien $B$, Serrière-Lanneau V, et al. ATP release after partial hepatectomy regulates liver regeneration in the rat. J Hepatol $2010 ; 52: 54-62$

38. Magnino F, St-Pierre M, Lüthi M, et al. Expression of intracellular calcium channels and pumps after partial hepatectomy in rat. Mol Cell Biol Res Commun $2000 ; 3: 374-9$

39. Nicou A, Serrière V, Hilly $M$, et al. Remodelling of calcium signalling during liver regeneration in the rat. J Hepatol 2007 ; $46: 247-56$.

40. Lagoudakis L, Garcin I, Julien B, et al. Cytosolic calcium regulates liver regeneration in the rat. Hepatology $2010 ; 52: 602-11$.

41. Lacampagne A, Fauconnier J, Richard S. Récepteur de la ryanodine et dysfonctionnement myocardique. Med Sci (Paris) 2008 ; 24 : 399-405.

\section{TIRÉS À PART}

G. Dupont
La question de la reproduction sexuée est cruciale pour appréhender les mécanismes de la perpétuation et de l'évolution de la majorité des espèces eucaryotes. Dans de nombreux cas, le genre est défini au niveau chromosomique et gouverne les modalités du brassage génétique dans les populations et les espèces. Le succès évolutif de la reproduction sexuée repose à la fois sur la différenciation des sexes mais aussi sur leur compatibilité / complémentarité. De cette dernière propriété s'ensuivent les phénomènes bien connus de sélection sexuelle des mâles par les femelles, l'exemple emblématique étant la sélection de la queue en roue chamarrée du paon mâle. Les raisons de la prédominance de la reproduction sexuée dans la nature et sa persistance au cours de l'évolution demeurent néanmoins mal évaluées.

Tous ces domaines d'étude sont actuellement révolutionnés par les progrès technologiques de la génomique tels que le séquençage haut débit et les nouvelles méthodes d'analyse globale (RNAseq, cartographie génomique globale des nucléosomes...). Des analyses phylogénétiques des analyses de génotypage de populations, et des analyses de typage épigénétique peuvent être maintenant conduites à l'échelle des génomes entiers. Elles produisent des résultats qui bouleversent déjà les raisonnements en vigueur sur l'évolution des éléments de régulation en cis et en trans des gènes.

L'objectif du colloque est donc de faire le point sur les avancées récentes en couvrant une grande diversité d'eucaryotes et en intégrant différents niveaux d'analyse, génétiques, épigénétiques, de la cellule à la population en passant par l'organisme. Notre ambition est de réunir des communautés scientifiques qui ont rarement l'occasion d'échanger et de confronter leurs points de vue, dans le but de promouvoir les interactions et collaborations et de permettre l'émergence de modèles intégratifs

Information et inscription: www.sfgenetique.org
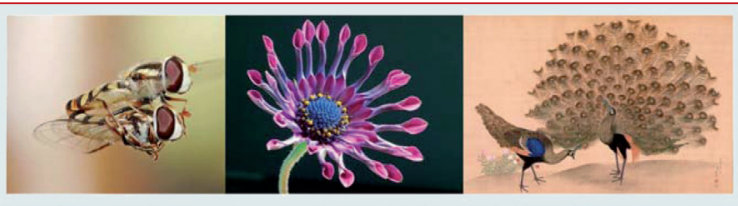

Genetics, Epigenetics and Evolution of Sex Chromosomes 9-10 June 2011 Université Paris-Diderot, Paris
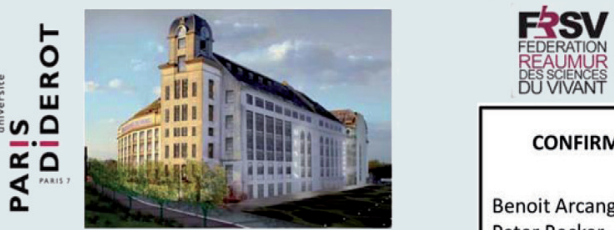

REAUMUR
DESSCINCES
DUVIVANT

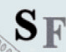

CONFIRMED SPEAKERS

Benoit Arcangioli

Peter Becker

Abdlehafid Bendahmane

The question of reproduction and sex is central to the evolution of species. A wealth of mechanisms, including genetic and epigenetic regulations, controls sexual identity. The recent progress in our understanding of the mechanisms underlying sexual definition/reproduction together with the increasing number of fully sequenced eukaryote genomes are expected to provide new insights to these questions.

The aims of the upcoming meeting "Genetics, Epigenetics The aims of the upcoming meeting "Cenetics, Epigenetics bre Evelution of sex Chromosome breakthroughs from eukaryotes including fungl, plants, insects and other invertebrates, fishes, birds and mammals, ditions between scientists from distinct fields but related topics of research.

\section{SESSIONS WILL COVER:}

Evolution

Sex determination

Chromosome organization

Recombination/Meiosis

Dosage compensation

Epigenetics

Brian Charlesworth

Cécile Fairhead

Tatiana Giraud

Jennifer Grave

Edith Heard

Ken McElreavey

Eric Meyer

Catherine Montchamp-Moreau

Claire Rougeulle

James Turner

Lucas Sanchez

Jean-Nicolas Volf

Denise Zickler

B. Arcangioli, G. Baldacci, C. Fairhead, C. Isnard,

C. Montchamp Moreau, C. Rougeulle

C. Montchamp Moreau,

contact@sfgenetique.org
More to follow..

caviesan 